Relations industrielles

Industrial Relations

\title{
Le syndicalisme des cadres
}

\section{Jacques Cousineau}

Volume 15, numéro 4, octobre 1960

URI : https://id.erudit.org/iderudit/1021945ar

DOI : https://doi.org/10.7202/1021945ar

Aller au sommaire du numéro

\section{Éditeur(s)}

Département des relations industrielles de l’Université Laval

ISSN

0034-379X (imprimé)

1703-8138 (numérique)

Découvrir la revue

Citer ce document

Cousineau, J. (1960). Le syndicalisme des cadres. Relations industrielles / Industrial Relations, 15(4), 492-495. https://doi.org/10.7202/1021945ar

Tous droits réservés @ Département des relations industrielles de l’Université Laval, 1960
Ce document est protégé par la loi sur le droit d'auteur. L'utilisation des services d'Érudit (y compris la reproduction) est assujettie à sa politique d'utilisation que vous pouvez consulter en ligne.

https://apropos.erudit.org/fr/usagers/politique-dutilisation/ 


\section{Conclusion}

Il n'y a pas en soi de modes de collaboration valables dans tous les pays et pour tous les groupes organisés. L'expérience révèle que, pour être efficace, un cadre de collaboration ne peut s'établir à partir d'un schème purement théorique. Il doit reposer sur des structures et des expériences qui rendent justice à la réalité des faits. Mème si les rapports de collaboration dans divers pays sont en vue d'objectifs qui, dans l'ensemble, s'apparentent, il n'y a nul lieu de s'étonner que chaque pays présente un visage qui lui soit propre.

\section{LE SYNDICALISME DES CADRES}

\section{JaCQues Cousineau, s.J.}

L'importance croissante de la place qu'occupent les travailleurs non manuels dans la main-d'oeuvre des pays industriels est un des aspects les plus significatifs de l'histoire économique et sociale du XXe siècle. Cet accroissement du nombre des travailleurs non manuels est à la fois absolu et relatif. Il résulte des besoins créés par le développement économique et social; de par le monde il y a une aspiration profonde à ce que tous les groupes sociaux aient des possibilités plus grandes d'épanouissement et un accès plus large au domaine culturel et dès lors bénéficient d'une élévation progressive des niveaux de vie.

Des enquêtes récentes faites notamment sur les répercussions de l'automation permettent de conclure que l'extraordinaire développement du progrès technique et la mise sur pied de puissants ensembles industriels ont entraîné, par suite des immenses concentrations d'hommes et de moyens matériels, la multiplication des professions de techniciens et d'ingénieurs, de mème que la croissance des fonctions d'encadrement dans l'entreprise.

\section{DÉFINITION DES CADRES}

Le terme «cadre » nous vient de France où il est employé couramment depuis une vingtaine d'années. La réalité qu'il représente ne se retrouve pas exactement dans les autres pays d'Europe ni dans l'entreprise nord-américaine, bien que la réalité décrite sous le nom de supervisor s'en approche singulièrement.

De façon générale, on entend par «cadres» le groupe des personnes qui dans une entreprise «encadrent» le personnel des ouvriers même qualifiés et des employés subalternes ou inférieurs; ils «encadrent» au sens de donner de la consistance et de l'animation à l'ensemble à cause de la part d'autorité qu'ils détiennent ou de leurs qualifications techniques ou intellectuelles.

M. Jacquin, dont la recherche sur les cadres du commerce et de l'industrie fait autorité, groupe en une définition fonctionnelle les divers éléments que son optique lui a permis d'entrevoir:

«Le cadre se caractérise essentiellement comme le salarié responsable de l'appréciation et de la mise en oeuvre de tout ou partie de la politique de l'entreprise. » 
Le cadre fait donc preuve d'initiative dans le choix des moyens et de leur mise en oeuvre, ce qui n'implique pas obligatoirement commandement. Il peut discuter les politiques et recommander des modifications, mais il faut retenir qu'apprécier ne signifie pas établir.

Dans l'entreprise nord-américaine, il y a des cadres, même si le terme français n'a pas son équivalent anglais. Au bas de l'échelon hiérarchique, mais en faisant partie, se trouve le contremaître, considéré dans la plupart des usines et par les législations tant étatsunienne que canadienne, comme un représentant essentiel de la direction. Au haut de l'échelon, immédiatement au-dessus de l'infra-structure qui comprend l'agencement de toutes les fonctions de l'entreprise, qu'on les répartisse selon les divisions classiques d'autorité ( «line») ou de conseil (《staff 》), se trouve l'organe supérieur de gestion, le conseil d'administration. Seul ce conseil possède l'autorité finale d'approuver ou de rejeter les politiques de l'entreprise. Il s'ensuit que tous les employés supérieurs qui ne siègent pas à ce conseil d'administration ne font que proposer, apprécier, formuler et faire appliquer des politiques. Leur rôle les classe parmi les cadres, supérieurs, intermédiaires ou inférieurs, selon la portée de leur action, à condition toutefois qui'ls soient des salariés proprement dits.

\section{DROTT SYNDICAL}

Ces employés supérieurs ou cadres, qui ne sont ni des employeurs ni des employés subalternes, ont-ils le droit de se syndiquer?

Au regard de la doctrine sociale de PEglise et de ceux qui la prônent avec autorité, la réponse ne semble pas hésiter vers l'affirmative.

S.S. Pie XII, le 27 novembre 1955, recevait en audience un important groupe de la Confédération italienne des dirigeants d'entreprise et de la Confédération internationale des Cadres. A cette occasion, le maître suprême de la pensée sociale chrétienne adressait un message:

« Nous désirons vous exhorter à apporter de plus en plus votre contribution à la solution du problème social, qui continue à laisser dans l'anxiété une grande multitude d'hommes.

Afin que le heurt des classes en opposition soit vraiment contenu; afin que tout le monde s'engage avec confiance et résolution dans la voie qui conduit au but souhaité, c'est-à-dire, à une coopération sincère et dynamique de toutes les forces vives de la nation pour le bien commun; afin que la doctrine sociale de l'Eglise soit reconnue et appliquée comme vraiment capable de détruire les racines qui donnent naissance à la haine et entretiennent la lutte des classes, vous avez été appelés, chers fils des Cadres, à donner votre contribution... Vous êtes donc dans la position idéale pour contribuer efficacement à l'instauration de ce régime de collaboration entre les classes sociales, qui est tant désiré par toutes les personnes honnêtes.

Ne vous épargnez pas, chers fils, dans cette oeuvre de pacification faite au nom de la justice et de l'amour. »

Elevé à cette heuteur, le syndicalisme ne demeure plus un droit, mais devient un devoir, l'accomplissement d'une haute mission sociale et d'un authentique apostolat.

Nos évêques du Québec n'avaient pas dit autre chose dans leur Lettre pastorale collective sur le Problème ouvrier de 1950. Le Code social de l'Union inter- 
nationale de Malines, qui constitue en dehors du Magistère de l'Eglise, une des synthèses les plus autorisées de la doctrine sociale catholique, a abordé nettement le problème de l'organisation syndicale des employés qui font déjà partie de professions.

D'ailleurs, sur le plan du droit international, il se constitue un corps de doctrine de plus en plus précis sur les relations humaines et celles du travail en particulier. L'article 23 (4) de la Déclaration universelle des droits de l'homme déclare explicitement que «toute personne a le droit de fonder avec d'autres des syndicats et de s'affilier à des syndicats pour la défense de ses intérêts $»$. Le problème de la protection du droit syndical et du droit d'organisation et de négociation collective, propre aux travailleurs non manuels, y compris le personnel technique, les cadres, etc., se pose désormais à l'échelle internationale.

La législation nationale varie énormément de pays en pays. Au Canada on peut dire, en résumé, que les employés supérieurs du Canada sont actuellement dans la situation même où se trouvaient les employés subalternes avant 1944. Tant dans notre législation fédérale que dans celle du Québec, aucun article de la loi ne peut être invoqué pour contraindre un employeur à reconnaître un syndicat de cadres comme agent de négociation. Le droit syndical des employés supérieurs ou cadres n'est donc pas protégé dans nos textes législatifs, inspirés du modèle étatsunien. Rien n'empêche toutefois un employeur de négocier avec ses employés supérieurs, comme autrefois avant 1944 des milliers d'employeurs éclairés négociaient avec leurs employés subalternes, sans aucune contrainte légale.

\section{AtTtTUdes CONCRÈTES}

Il faut reconnaître que peu d'employeurs le font et peu d'employés supérieurs de chez nous expriment ouvertement leur désir et prennent la peine ou le risque de se grouper en vue de négocier collectivement. Il ne suffit pas que les négociations collectives soient autorisées, sinon favorisées, par la législation, pour qu'elles aboutissent; il faut aussi qu'employeurs et employés soient disposés à recourir à la procédure établie ou, comme dans le cas présent, à établir d'un commun accord. Avant de s'entendre sur les méthodes, il importe que les parties intéressées considèrent le syndicalisme et les négociations collectives comme souhaitables et utiles en soi en ce qu'elles permettent de se mettre d'accord sur les conditions de travail et d'établir des relations satisfaisantes.

Or c'est un fait que les professions non manuelles qui groupent le plus grand nombre de salariés sont mal organisées dans la plupart des pays; c'en est un autre que les syndicats de cadres représentent rarement plus qu'une faible minorité de ceux dont ils défendent les intérêts.

Cette faiblesse s'explique par des facteurs qui tiennent à la nature même du travail, à l'attitude des intéressés eux-mêmes et à celle de leurs employeurs. Pour être complet. il me faudrait analyser les conséquences (et donc les avantages et les inconvénients) que prévoient de part et d'autre les partisans et les opposants de la syndicalisation des cadres. 


\section{ABOUTHSBEMENT SOCIAL}

Il faudrait aussi toucher à l'implantation sociologique des cadres et rappeler les thèses qui s'affrontent au sujet de la nature sociale de la catégorie des cadres et de son rôle sur la vie sociale. Il y en a trois qui sont le plus fréquemment évoquées selon lesquelles les cadres sont présentés comme étant, soit une fraction du prolétariat - c'est la prétention marxiste - soit la classe dirigeante en puissance - c'est le propos technocratique - soit enfin le tiers parti entre capital et travail. La plupart des cadres toutefois se rallient à la conception du tiers parti selon laquelle ils constituent un groupe intermédiaire entre le capital et le travail et forment le noyau des classes dites moyennes.

Mais le syndicalisme de cadres a-t-il une mission? On acceptera d'emblée, j'espère, l'idée qu'à l'époque où se redécouvre le sens de la communauté, les cadres peuvent moins que jamais s'isoler de la vie économique et sociale. Mais, dans ce contexte social, l'organisation syndicale des employés supérieurs a-t-elle mission au regard de la doctrine sociale de l'Eglise?

De fait, si l'on considère l'aspect constructif du syndicalisme, il faut dire que les cadres ont dans l'organisation syndicale un moyen institutionnel privilégié pour mettre en oeuvre leurs responsabilités sociales, économiques et, jusquà un certain degré, civiques, tant au plan national et international, qu'au plan de la profession et de l'entreprise.

Pour justifier ces affirmations, je devrais reprendre le problème de plus haut et parler de la lutte pour la justice comme une des exigences essentielles de la foi et une des missions du chrétien dans le monde; je devrais ensuite poser les deux questions complémentaires: 1) Notre société actuelle a-t-elle atteint un état de justice auquel il n'y a plus rien à ajouter; 2) Le cadre, ce haut fonctionnaire de l'économie moderne, dispose-t-il d'un certain pouvoir pour la promotion de la justice dans la vie sociale?

La réponse va de soi. En dépit des problèmes qu'il pose et quelles que soient ses modalités d'organisation, le syndicalisme des cadres est souhaitable à notre époque de multiplication des institutions et d'accélération des transformations sociales, époque qui exige de nous le courage de l'intelligence, l'attention aux problèmes nouveaux et surtout aux nouvelles formes de l'oppression des hommes. Le complice majeur de l'injustice en notre temps d'évolution rapide, c'est la myopie sociale qui consiste à limiter son horizon à sa spécialisation ou à ses intérêts individuels. Il faut aujourd'hui une mobilisation plus large et plus profonde des énergies parce que la réalité économique et sociale est faite d'une multitude de micro-décisions quotidiennes. Les corps sociaux, tels les syndicats, qui sont plus que la somme des pouvoirs de décision individuels, infléchissent la marche des événements et peuvent acheminer vers un meilleur état de justice sociale. Ils sont d'autant plus bienfaisants que leurs membres sont animés de l'esprit qu'anime l'Eglise dans son éternel combat pour la justice. 